EXTENDED REPORT

\title{
Secondary retinal changes associated with choroidal naevi and melanomas documented by optical coherence tomography
}

\author{
S Muscat, S Parks, E Kemp, D Keating
}

Br J Ophthalmol 2004;88:120-124

See end of article for authors' affiliations

Correspondence to: Dr David Keating, Electrodiagnostic Imaging Unit, Tennent Institute of Ophthalmology, Gartnavel General Hospital, 1053 Great Western Road, Glasgow G12 OYN, UK; d.keating@ clinmed.gla.ac.uk

Accepted for publication 28 April 2003
Aims: To establish the characteristics of secondary retinal changes associated with the presence of choroidal melanomas and choroidal naevi as documented by optical coherence tomography (OCT). Methods: Twenty patients with untreated choroidal melanoma and 40 patients with presumed choroidal naevi managed by regular observation were included in the study. OCT scans across the surface of the tumour and surrounding tissue were acquired for all participants. The appearance of retinal tissue on the OCT scans was considered to be abnormal if it did not display the well defined band structure characteristic of normal tissue on OCT scans.

Results: Serous retinal detachments were observed in all patients with choroidal melanoma and 18 out of 20 also had abnormal retinal structure or intra-retinal splitting in the tissue overlying the tumour. Out of the 40 patients with presumed choroidal naevi, 12 had serous detachments and three had either abnormal retinal structure or intra-retinal splitting overlying the lesion.

Conclusions: Secondary retinal changes associated with choroidal lesions can be documented by OCT. These changes were observed in most patients with choroidal melanoma included in the study group but were far less prevalent in the patients with presumed choroidal naevi. OCT is also able to identify the presence of small serous detachments before they become clinically visible. Follow up of these patients is required to determine whether the OCT results may be of use in the differential diagnosis of small choroidal lesions.
C linical and histopathological studies on choroidal melanomas have shown a number of associated secondary changes in the overlying retina and retinal pigment epithelium (RPE) and these are well documented in the literature. ${ }^{12}$ Some choroidal naevi have also been known to cause similar changes. ${ }^{134}$ It has been suggested that careful observation of secondary changes associated with choroidal lesions may be important in the differential diagnosis of choroidal lesions. ${ }^{15}$ At present, clinical assessment of choroidal lesions is generally carried out using biomicroscopy, fundus photography, and ultrasound.

Optical coherence tomography (OCT) is an imaging technique that gives cross sectional information on retinal and pigment epithelial structure with a resolution of around $10-15 \mu \mathrm{m} .{ }^{67}$ OCT has been used successfully in a variety of clinical situations ${ }^{8-11}$ and studies have shown that prototype and commercially available OCT scanners show a high degree of repeatability and reproducibility. ${ }^{12-16}$ A study by Schaudig et $a l^{17}$ attempted to obtain in vivo information about the histological nature of 22 cases of choroidal melanoma using OCT. The authors of this study concluded that since the OCT beam is strongly scattered by the RPE, reflections from within the tumour mass are weak and therefore OCT is not useful for in vivo histological typing of choroidal tumours. However they found that changes of the RPE and the neurosensory retina in patients with choroidal melanoma were well visualised by OCT. This study only included patients with large malignant choroidal melanomas, some of which had been treated, and did not consider patients with smaller melanomas or naevi. To date there does not appear to have been any study that assesses the differences in retinal changes associated with malignant choroidal melanomas and presumed choroidal naevi using OCT. The aim of this study was therefore to establish the characteristic retinal changes, as visualised by OCT, in patients with choroidal naevi or choroidal melanomas of various sizes.

\section{MATERIALS AND METHODS Study group}

The study was carried out prospectively on a total of 60 patients attending the Ocular Oncology Service at the Tennent Institute over a period of 36 months. The inclusion criteria were the presence of a choroidal lesion which was untreated at the time of OCT scanning and located such that OCT scans around its base and across its surface could be easily acquired, no history of other conditions which could affect retinal structure and clear media to ensure good quality OCT scans. Patients were excluded if they had a choroidal melanoma which had previously been treated in any way as treatment may have affected retinal structure; if they suffered from any other condition which could affect retinal structure; if they had non-clear media which would reduce the quality of the OCT scans, or if they had very peripheral lesions which could not be imaged using OCT. Ethics committee approval for this study was obtained and all participating patients gave their full consent after the nature and aims of the study had been fully explained to them.

All patients included in the study underwent wide-field binocular fundal examination as well as ultrasound assessment. The location of the tumour, basal diameter, and tumour thickness were recorded. Measurement of tumour thickness was made from the ultrasound scans which were assessed by an experienced radiologist. Tumours were classified as choroidal melanomas or naevi on the basis of these clinical findings, mainly size, with tumours under $1 \mathrm{~mm}$ thick being classified as naevi and those over $3 \mathrm{~mm}$ being diagnosed as choroidal melanoma. Some tumours under $3 \mathrm{~mm}$ were classified as choroidal melanomas following 
documented growth during the observation period. All tumours diagnosed as choroidal melanoma were treated whereas those classified as naevi were kept under regular observation for any signs of growth. Several clinical factors have been associated with increased risk of growth in small choroidal tumours. Studies have shown that tumour thickness greater than $2 \mathrm{~mm}$, the presence of sub-retinal fluid, visual symptoms, orange pigmentation, proximity to the optic disc, and the absence of drusen or RPE atrophy are factors which may be predictive of growth. ${ }^{18-20}$ A record of any of these features noticed during clinical examination was also kept for both categories of tumours.

\section{Scanning protocol}

The scanner used in this study was the commercially available OCT 2 scanner (Humphrey Instruments, San Leonardo, CA). Scanning was performed using a superluminescent diode operating with a wavelength of $840 \mathrm{~nm}$ and maximum power of $750 \mu \mathrm{W}$. Images were displayed as a pseudo-colour plot where different colours represented differences in the reflective properties of the retinal tissue.

No formal scanning protocol was designed for this study as the sizes and locations of tumours varied considerably between patients, making it impossible to design a scanning protocol that would be applicable to all cases. The longitudinal depth of an A-scan acquired by the OCT scanner is $3 \mathrm{~mm}$ in air, approximately $2 \mathrm{~mm}$ in retinal tissue, meaning that any structures separated by more than $2 \mathrm{~mm}$ in the longitudinal direction could not be seen entirely on a single scan. In the case of lesions under $2 \mathrm{~mm}$ high, we attempted to acquire at least one scan which extended from a flat, unaffected portion of the retina to the highest point of the lesion that could be imaged. For larger lesions it was not possible to have both the flat portion of the retina close to the base of the lesion and the surface of the lesion in focus at the same time. In these cases we first acquired scans around the base of the lesion, then re-adjusted the focussing control until a clear image of the surface of the lesion was visible on the fundus monitor and acquired a few scans across the surface of the lesion. All scanning and analysis of scans was performed by one of the authors (SM).

\section{Scan analysis}

The characteristics of OCT scans in the normal population are well established. ${ }^{61-16}$ OCT scans of normal tissue show two well defined highly reflecting red and white bands which are thought to correspond to the nerve fibre layer (NFL) and the RPE-choriocapillaris complex. These bands are separated by a region of lower reflectivity, thought to correspond to the mid and outer retinal layers. Scans in our study were analysed to assess whether the appearance of retinal tissue around or overlying the lesion differed from the normal appearance of retinal tissue on OCT scans. Scans across the surface of the tumour and around its base revealed three distinct patterns of abnormalities-serous retinal detachments around and overlying the tumour, intra-retinal splitting, or cystic spaces in the overlying retina and loss of normal retinal architecture overlying the tumour. A serous retinal detachment was identified as an area of low reflectance that did not cause shadowing of underlying structures and was situated immediately anterior to the RPE-choriocapillaris band, which was still well defined. Typical examples are shown in Figures 1A and B. Intra-retinal splitting and cystic spaces were identified as regions of low reflectance within the neurosensory retina as shown in figure $1 C$. In these cases, the bands corresponding to the NFL and RPE-choriocapillaris complex were still well defined. Loss of normal retinal architecture was identified in cases where the retinal tissue overlying the tumour no longer showed the characteristic
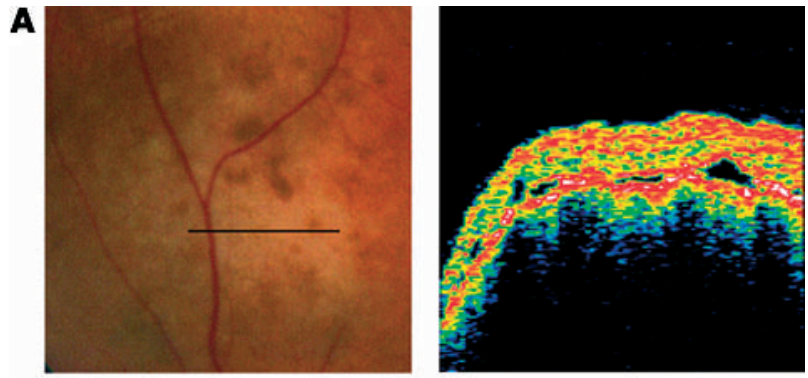

B
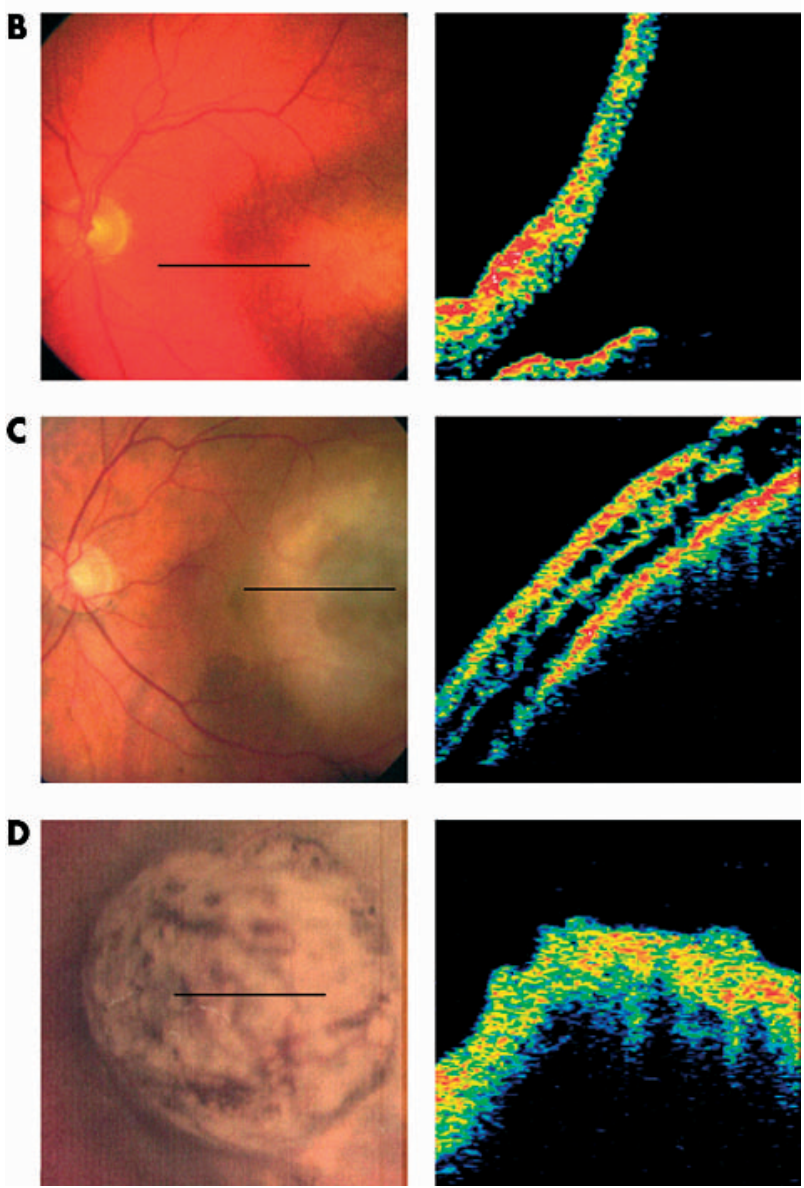

Figure 1 Typical secondary retinal changes associated with choroidal melanomas-OCT scans and corresponding fundus images are shown. The line on the fundus images corresponds to the location of the OCT scan. (A) Scan across the surface of a choroidal melanoma showing small serous detachments of the retina overlying the tumour. (B) Serous detachment of the retina at the base of a choroidal melanoma. (C) Intraretinal splitting overlying the tumour. (D) Highly abnormal structure of retinal tissue overlying a choroidal melanoma.

appearance of retinal tissue on OCT. Scans that no longer showed this well defined layer structure were considered to be abnormal. In these cases, the band corresponding to the RPE-choriocapillaris complex could no longer be identified. Figure 1D shows an example of loss of normal retinal architecture-scans across the surface of the tumour showed a structure less highly reflecting signal.

We did not attempt to make any measurements from the OCT scans. Measuring the height of the lesion from OCT seemed unnecessary as this information was available from conventional ultrasound, which was performed on all patients attending the clinic. Moreover, as explained above, for lesions thicker than $2 \mathrm{~mm}$, it was impossible to have both the base and the surface of the tumour on the same scan. 

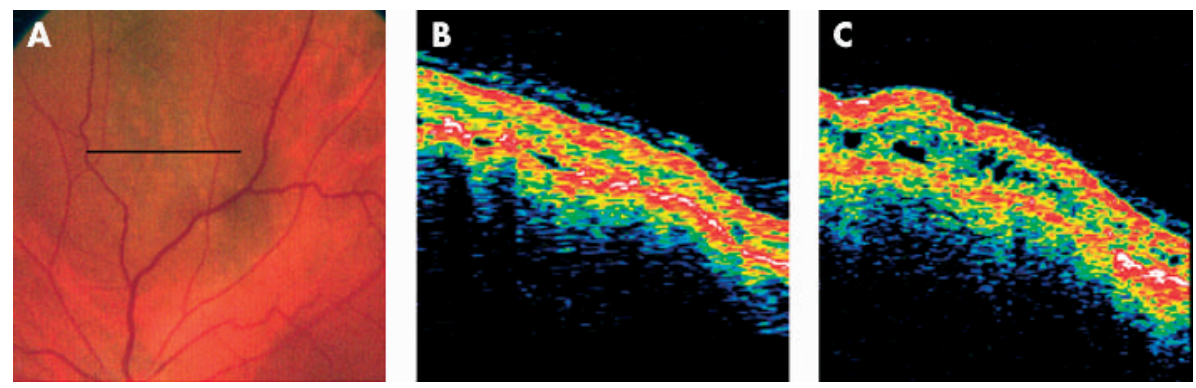

Figure 2 OCT scans of a tumour that enlarged during the observation period. (A) Fundus image showing the location of the OCT scans. (B) Scan across the tumour surface showing a few small serous detachments but normal retinal architecture. (C) Scan of the same area obtained 15 months after presentation, after tumour growth had been recorded. Cystoid spaces can now be seen over the tumour surface.

Therefore, measuring the height of larger lesions from OCT was not possible. Schaudig et $a l^{17}$ made measurements of retinal thickness across the surface of the tumour. However, we found that in a number of cases, the retina overlying the tumour no longer had the appearance of normal retinal structure and different layers such as the RPE and NFL could no longer be identified. This made it impossible to determine the positions of the retinal boundaries and to make accurate measurements of retinal thickness.

\section{RESULTS}

Sixty patients, 34 male and 26 female, were included in the study. Ages ranged from 31 to 95 years (mean 65.9 (SD 11.7) years). Out of these 60 cases, 20 tumours were classified as malignant choroidal melanoma and 40 were classified as naevi.

\section{Patients with choroidal melanoma}

Fourteen of these 20 patients were diagnosed with malignant choroidal melanoma at their first visit to this centre and they proceeded to immediate treatment. The tumour thickness in this group of 14 patients ranged from 3 to $7.3 \mathrm{~mm}$ (mean 5.6 (SD 1.6) $\mathrm{mm}$ ) and basal diameter ranged from 5 to $15 \mathrm{~mm}$ (mean 10.7 (SD 3.3) mm). On clinical examination, eight of these patients were found to have associated serous detachments, 13 had visual symptoms, eight had orange pigmentation over the tumour surface, and none had drusen.

The other six patients with choroidal melanoma were diagnosed following documented growth or changes in their lesions. Tumour thickness and basal diameter at diagnosis ranged from 1.4 to $5.4 \mathrm{~mm}$ (mean 3.1 (SD 1.4) $\mathrm{mm}$ ) and from 6 to $14.9 \mathrm{~mm}$ (mean 9.9 (SD 3.5 ) $\mathrm{mm}$ ) respectively. The average increase in tumour thickness during the observation period was $1.7 \mathrm{~mm}$. OCT scans were performed on more than one occasion during follow up for four of these patients. In the other two cases, this was not possible since the patients had been initially managed at other centres and were only referred to our centre after changes in the lesion were observed. At the time of diagnosis, four had serous detachments, four had visual symptoms, three had orange pigmentation over the tumour surface and five had no drusen.

Nine of the 20 patients with choroidal melanoma were treated with ruthenium plaque only, another four received proton beam therapy only, and another four had local resection followed by Ruthenium plaque brachytherapy. Three patients, two who had received proton beam therapy and another who had been treated with Ruthenium plaque, had to undergo enucleation of the eye because of further growth of the tumour despite the initial treatment. Histological analysis of the tumour was therefore available in seven cases. In all cases, the diagnosis of malignant melanoma was confirmed by histological analysis. Five tumours were of mixed cell type (epitheloid and spindle-B) and two were of Spindle-B type.

OCT showed serous retinal detachments surrounding or overlying the tumour in all 20 cases, including eight cases in which no serous detachments had been noted on clinical examination. Only two patients had no evidence of intraretinal splitting or abnormal retinal structure in the tissue overlying the tumour. All of the other 18 patients had at least one of these abnormalities. Eight had only intra-retinal splitting, seven had only abnormal structure, and three had abnormal retinal structure with low reflectance areas within, suggestive of cystic spaces.

The repeat scans from one patient who was diagnosed with choroidal melanoma 15 months after initial presentation, following enlargement of the lesion from $1 \mathrm{~mm}$ to $2 \mathrm{~mm}$, are interesting. The initial scan across the surface of the lesion showed a raised, but essentially normal retinal structure with a few very small serous detachments (fig 2B). Repeat scans of the same region were acquired at a follow up visit 15 months later. These scans were made using the Repeat Scan option and the corresponding fundus pictures were analysed to ensure that the same regions had been scanned on both occasions. These scans (Fig 2C) showed cystoid spaces which had not been evident from the first set of scans. Repeat scans from the other patients whose naevi showed growth during the period of observation were acquired at shorter intervals and showed no appreciable inter-visit differences other than an increase in elevation.

In cases where OCT identified subclinical serous detachments of the retina, the maximum separation between neurosensory retina and the RPE-choriocapillaris complex was measured from the OCT scans. These separations ranged from 15 to $120 \mu \mathrm{m}$ (mean 75.3 (SD 23.8) $\mu \mathrm{m}$ ).

\section{Patients with presumed naevi under observation}

The remaining 40 patients in this study currently have a diagnosis of choroidal naevus and are being managed by regular observation. Tumour thickness ranged from $0 \mathrm{~mm}$ (flat) to $2.5 \mathrm{~mm}$ (mean 0.8 (SD 0.8$) \mathrm{mm}$ ) and basal diameter ranged from 2 to $7.5 \mathrm{~mm}$ (mean 5.1 (SD 1.8 ) $\mathrm{mm}$ ). The number of risk factors associated with increased risk of growth was calculated for each patient. Seven patients (17.5\%) had lesions over $2 \mathrm{~mm}$ in thickness, $12(30 \%)$ had lesions within 1 disc diameter of the optic disc, one $(2.5 \%)$ had clinically detected subretinal fluid associated with the lesion, five $(12.5 \%)$ had associated visual symptoms, 13 $(32.5 \%)$ had no drusen, and five $(12.5 \%)$ had orange pigmentation within the lesion. Two patients $(5 \%)$ had six risk factors, two $(5 \%)$ had five, one $(2.5 \%)$ had four, three (7.5\%) had three, seven (17.5\%) had two, 11 (27.5\%) had one, and the remaining 14 (35\%) had none of the risk factors associated with increased risk of tumour growth. 
Scans from these 40 patients were analysed for changes similar to those observed in the melanoma group. Twenty seven patients $(67.5 \%)$ showed none of these abnormalitiesno serous detachments at the base of the lesion or in the retinal tissue overlying the lesion, no intra-retinal splitting, and no structural abnormalities. Out of the remaining 13 $(32.5 \%), 12$ had serous retinal detachments. Serous detachments had been noticed clinically in only one of these patients. The separation between neurosensory retina and underlying RPE-choriocapillaris in these cases of subclinical serous detachments was similar to that observed in the melanoma group ranging from 17 from $118 \mu$ m (mean 68.2 (SD 26.6) $\mu \mathrm{m}$ ). Two patients were found to have intra-retinal splitting in the tissue overlying the lesion (fig 3A). Changes in the retinal structure, similar to those found in the melanoma group, were observed in one patient (fig 3B).

\section{DISCUSSION}

It is known that malignant choroidal melanomas and choroidal naevi may cause secondary retinal changes. ${ }^{12}$ To date, there is little information on the OCT appearance of these secondary changes and the aim of this study was therefore to establish the characteristic appearance of secondary retinal changes associated with choroidal melanomas and naevi when imaged with OCT.

Clinical and histopathological studies on patients with choroidal tumours have shown that common associated secondary changes include serous retinal detachment extending beyond the limit of the tumour, atrophy of the RPE, atrophy of the retina starting in the photoreceptor layer, cystoid retinal degeneration leading to retinoschisis, and invasion of the sensory retina by the tumour. ${ }^{12}$ Our study showed that serous detachments, cystoid retinal degeneration and retinoschisis could be visualised in vivo using OCT. In some patients, the retina overlying the tumour appeared to have lost the characteristic band structure associated with normal retinal tissue on OCT. Since the RPE-choriocapillaris layer could not be identified in these patients, it is possible that atrophy of the RPE had occurred. This study shows that
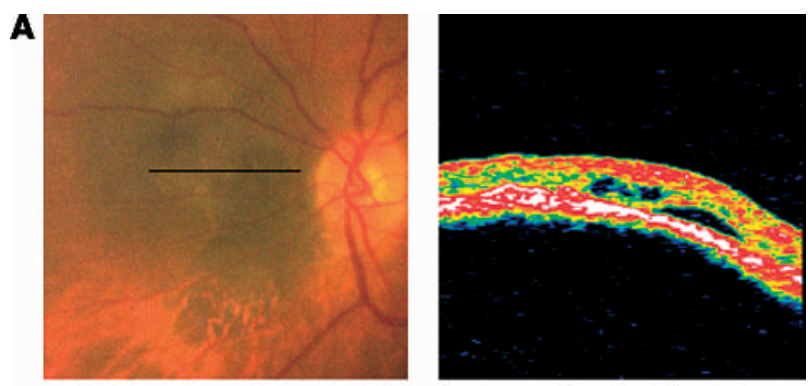

B
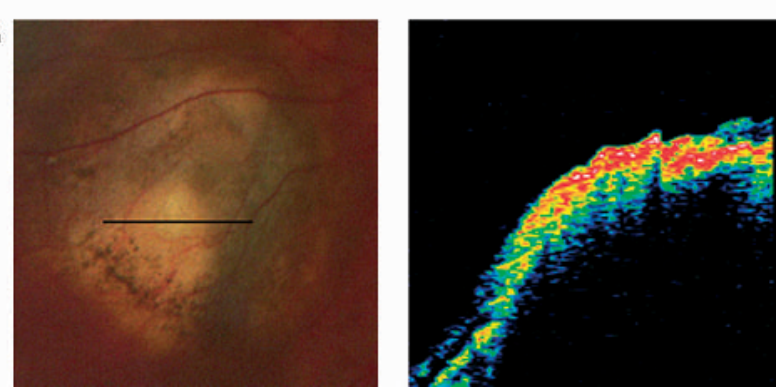

Figure 3 Secondary retinal changes in patients with naevi. OCT scans and corresponding fundus images are shown. (A) Intra-retinal cystic spaces in a patient wisth a naevus. (B) Abnormal retinal structure overlying a naevus. although OCT in its current state cannot give any in vivo information on the histology of choroidal tumours, this imaging technique can clearly show retinal changes in the tissue surrounding and overlying choroidal tumour.

An interesting observation from our study was the fact that the majority of patients with diagnosed choroidal melanoma in our study group were found to have cystic degeneration, retinoschisis, or abnormal retinal structure suggestive of RPE or retinal atrophy. By contrast, only a small percentage of patients with presumed naevi showed these changes. Out of the three patients with presumed naevi who did have these changes, two have been under observation for less than two years and have some of the risk factors associated with increased risk for growth, so the possibility that these are in fact small choroidal tumours cannot be ruled out at this stage. The third patient has been under observation for over 30 years but has recently developed visual symptoms in the affected eye. Therefore it is possible that the lesion in this case was a dormant melanoma that has recently become active.

The case presented in figure 2 shows repeat OCT scans of a lesion that grew from just under $1 \mathrm{~mm}$ at first presentation to $2 \mathrm{~mm}$ during a 15 month period. At presentation, the lesion was classified as suspicious due to its proximity to the optic disc, the presence of subretinal fluid accumulation at the macula, visual symptoms, absence of drusen, and clumps of orange pigmentation over the tumour surface. It was therefore decided to re-examine the lesion at frequent intervals. The initial scans (fig 2B) showed an elevated area over which the retinal structure appeared relatively intact. There were a few small regions of subretinal fluid accumulation, but both the nerve fibre layer and retinal pigment epithelium layer were still well defined. The follow up scans acquired 15 months later showed cystic spaces within the retina overlying the tumour. This cystic degeneration could have simply been a result of chronicity; however, given that similar cystic degeneration was observed in several patients with diagnosed choroidal melanoma but in very few of those with naevi some of which were longstanding, it is possible that the development of cystic spaces in this particular patient was caused by the probable malignant nature of this tumour. This particular case indicates that OCT can document the development of secondary retinal changes and could therefore be a useful tool in the follow up of patients with suspicious naevi.

The majority of serous retinal detachments observed on OCT scans of patients with presumed naevi had not been noticed clinically. As the presence of subretinal fluid has been associated with a greater risk of growth, ${ }^{18-20}$ this finding could be of value in the differential diagnosis of small choroidal lesions.

Investigation, using OCT, of patients with diagnosed choroidal melanoma has shown that the majority of these patients have associated secondary retinal changes. The incidence of similar changes in patients with presumed choroidal naevi appears to be much lower. Long term follow up of the patients with presumed naevi that show changes similar to those of patients with melanomas is required to establish whether these findings are of diagnostic value.

\section{ACKNOWLEDGEMENT}

This research was supported by Scottish Office grant K/MRS/50/ C2712.

\section{Authors' affiliations}

S Muscat, S Parks, E Kemp, D Keating, Tennent Institute of Ophthalmology, Gartnavel General Hospital, Glasgow, UK

\section{REFERENCES}

1 Damato BE, Foulds WS. Tumour-associated retinal pigment epitheliopathy. Eye 1990;4:382-7. 
2 Shields JA, Shields CL. Intra-ocular tumours: a text and atlas I. Pennsylvania: WB Saunders Company, 1992:117-36.

3 Shields JA, Shields CL. Intra-ocular tumours: a text and atlas. Pennsylvania: WB Saunders Company, 1992:85-100.

4 Gonder JR, Augsburger JJ, McCarthy EF, et al. Visual Loss associated with choroidal naevi. Ophthalmology 1982;89:961-5.

5 Gass JDM. Problems in the differential diagnosis of choroidal naevi and malignant melanomas. Am J Opthalmol 1977;83:299-323.

6 Huang D, Swanson EA, Lin CP, et al. Optical coherence tomography. Science 1991;254:1178-81.

7 Hee MR, Izatt JA, Swanson EA, et al. Optical coherence tomography for ophthalmic imaging. IEEE Eng Med Biol 1995;14:67-76.

8 Puliafito C, Hee MR, Schuman JS, et al. Optical coherence tomography of ocular diseases. New Jersey: Slack Incorporated, 1996:39-368.

9 Baumal CR. Clinical applications of optical coherence tomography. Curr Opin Ophthalmol 1999;10:182-8.

10 Puliafito CA, Hee MR, Lin CP, et al. Imaging of macular diseases with optical coherence tomography. Ophthalmology 1995;102:217-29.

11 Hee MR, Izatt JA, Swanson EA, et al. Optical coherence tomography of the human retina. Arch Ophthalmol 1995;113:325-32.

12 Baumann M, Gentile RC, Liebmann JM, et al. Reproducibility of retinal thickness measurements in normal eyes using optical coherence tomography. Ophthalmic Surg Lasers 1998;29:280-5.
13 Koozekanani D, Roberts $C$, Katz SE, et al. Intersession repeatability of macular thickness measurements with the Humphrey 2000 OCT. Invest Ophthalmol Vis Sci 2000;41:1486-91.

14 Muscat S, Parks S, Kemp E, et al. Repeatability and reproducibility of macular thickness measurements with the Humphrey OCT system. Invest Ophthalmol Vis Sci 2002;43:490-5.

15 Schuman JS, Pedut-Kloizman T, Hertzmark E, et al. Reproducibility of nerve fibre layer thickness measurement coherence tomography. Ophthalmology 1996;103:1889-98.

16 Blumenthal EZ, Williams JM, Weinreb RN, et al. Reproducibility of nerve fibre layer thickness measurements by use of optical coherence tomography. Ophthalmology 2000;107:2278-82.

17 Schaudig U, Hassenstein A, Bernd A, et al. Limitations of imaging choroidal tumours in vivo by optical coherence tomography. Graefe's Arch Clin Exp Ophthalmol 1998;236:588-92.

18 Shields CL, Cater J, Shields JA, et al. Combination of clinical factors predictive of growth of small choroidal melanocytic tumours. Arch Ophthalmol 2000;118:360-4

19 Shields CL, Shields JA, Kiratli H, et al. Risk factors for growth and metastasis of small choroidal melanocytic tumours. Trans Am Ophthalmol Soc 1995;93:259-75.

20 The Collaborative Ocular Melanoma Study Group. Factors predictive of growth and treatment of small choroidal melanoma. COMS Report no.5. Arch Ophthalmol 1997;115:1537-44.

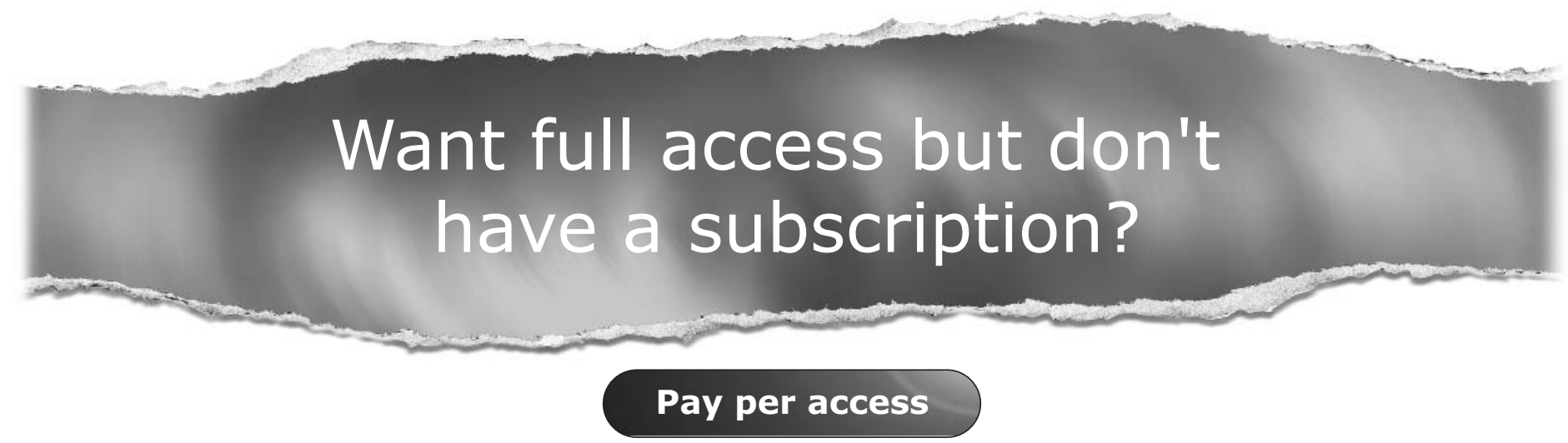

For just US\$25 you can have instant access to the whole website for 30 days. During this time you will be able to access the full text for all issues (including supplements) available. You will also be able to download and print any relevant pdf files for personal use, and take advantage of all the special features British Journal of Ophthalmology online has to offer.

\section{www.bjophthalmol.com}

